\title{
Social Connection is Essential in Long-Term Care Homes: Considerations During COVID-19 and Beyond
}

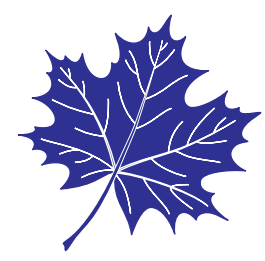

Jennifer Bethell, $\mathrm{PhD}^{1,2}$, Hannah M. O’Rourke, RN, $\mathrm{PhD}^{3}$, Heather Eagleson ${ }^{4}$, Daniel Gaetano ${ }^{4}$, Wayne Hykaway ${ }^{4}$, Carrie McAiney, PhD $^{5,6}$

${ }^{1}$ KITE-Toronto Rehabilitation Institute, University Health Network, Toronto, ON,

${ }^{2}$ Institute of Health Policy, Management and Evaluation, University of Toronto, Toronto, ON, ${ }^{3}$ Faculty of Nursing,

University of Alberta, Edmonton, AB, ${ }^{4}$ Canadian Consortium on Neurodegeneration in Aging (CCNA) Engagement

of People with Lived Experience of Dementia Advisory Group (https://ccna-ccnv.ca/contact), ${ }^{5}$ University of Waterloo,

Waterloo, ON, ${ }^{6}$ Schlegel-University of Waterloo Research Institute for Aging, Waterloo, ON, Canada

https://doi.org/10.5770/cgj.24.488

\begin{abstract}
COVID-19 has had a profound impact on long-term care (LTC) homes in Canada. But the measures put in place to control infection within LTC homes have also had devastating impacts on the health and well-being of residents through the effects on social connection. Here, we offer guiding principles to enable social connection and promote health and quality of life for LTC residents during COVID-19 and beyond. These principles were generated by a working group of the $\mathrm{CO}$ VID-19 and Dementia Task Force, convened by the Alzheimer Society of Canada to identify the urgent and emerging issues raised by COVID-19 for Canadians with dementia.
\end{abstract}

Key words: social network, social engagement, social support, loneliness, dementia, long-term care homes

The global pandemic of COVID-19 has challenged us all to fulfill our need for human connection-but LTC home residents and their families have been profoundly and disproportionately affected. Measures put in place to protect LTC residents from COVID-19 have included prohibiting visits from family and friends, and restricting activities and contact with other residents, staff, and care providers within the home. These measures have had unintended consequences on the social connections of LTC residents, resulting in isolation from loved ones and care partners, with little opportunity to engage in LTC home life. The benefits of infection control have come with serious risks to physical and mental health, and challenge the human rights of LTC residents and their families.

Here, we offer guiding principles to enable social connection and promote health and quality of life for LTC residents during COVID-19 and beyond. The principles were generated by a working group of the COVID-19 and Dementia Task Force, convened by the Alzheimer Society of Canada to identify the urgent and emerging issues raised by COVID-19 for Canadians with dementia. We highlight solutions that enhance social connection and improve care and support for people living with dementia in LTC homes, as well as their caregivers and families.

Social connection includes a number of separate but interrelated concepts. We considered the following aspects of social connection that matter to residents, family, and care staff in LTC homes: ${ }^{(1,2)}$

- Social Networks: the webs of social relationships that surround us and the characteristics of those ties;

- Social Support: the emotional, social, physical, and financial help we give to and get from others in our social network;

- Social Engagement: taking part in activities with others in our social network; and

- Social Connectedness: feelings of belonging and being cared for; the opposite to feeling lonely.

\section{Social Connection is a Basic Human Need}

We all deserve to feel cared about and have a sense of belonging; these are basic human needs. ${ }^{(3)}$ For residents of LTC homes, social connection is essential to quality of life. ${ }^{(4)}$ LTC home residents, $87 \%$ of whom experience cognitive impairment, ${ }^{(5)}$ can thrive when the need for social connection is met. ${ }^{(4)}$ Conversely, people with dementia have described how loneliness undermines quality of life; kind, loving, and respectful interactions with LTC staff, residents, and family and friends are all important to consider in promoting quality of life. ${ }^{(6)}$ Maintaining positive relationships with family and friends is of utmost importance to many 
LTC residents, and a lack of visits from family and friends can lead to feelings of abandonment. ${ }^{(6)}$ Both persons with dementia and care partners have described how symptoms of dementia, compounded by physical health issues as well as lack of understanding and stigma towards dementia, can limit social connection. ${ }^{(7)}$

\section{Social Connection Supports the Mental and Physical Health of LTC Residents}

Research studies conducted in LTC homes have demonstrated that social connection is linked to mental health outcomes including depression, cognitive decline, and behavioural symptoms of dementia. ${ }^{(8)}$ Similarly, studies have also shown social connection is associated with physical health outcomes for LTC residents, including mortality ${ }^{(9)}$ and self-rated health. (10) For residents of LTC homes, family and friends provide crucial social support, from hands-on assistance (e.g., during meals) to ensuring that staff understand who a resident is as a person and within a unique life history. ${ }^{(11)}$ Given the chronic understaffing in LTC homes, ${ }^{(12)}$ the impact of absent family and friends is felt throughout the care home; without the day-to-day support provided to LTC residents from their family and friends, already-stretched LTC staff must now further divide their time with residents, which may detract from quality of care. Taken together, the absence of social connection has negative impacts on the physical and mental health of LTC residents. LTC home administrators, staff, families, and residents are left to balance the risks, benefits, and human rights implications of the different visitor policy responses being implemented across jurisdictions. ${ }^{(13)}$

\section{Strategies that can Help Build and Maintain Social Connection for LTC Residents}

Building and maintaining social connection among LTC residents, and between residents and family and LTC staff, should be a key consideration, both during COVID-19 and beyond. Existing reviews have identified few studies that have rigorously tested the effectiveness of interventions in promoting social connection for people living in LTC homes, ${ }^{(14,15)}$ and even fewer have specifically targeted people with dementia. (3) However, qualitative studies with older adults have found that maintaining contact with others (in person or by phone or other means) and engaging in (even solitary) purposeful activity are key strategies to prevent loneliness. ${ }^{(3)} \mathrm{A}$ recent scoping review of published observational and intervention research conducted in LTC homes that assessed social connection outcomes ${ }^{(8)}$ highlighted 12 low-cost strategies that could be adapted for COVID-19 or other disease outbreaks. These strategies can be implemented right now to help build and maintain social connections with LTC residents:

1. Manage pain (e.g., ask about pain and consider treating with medication and/or non-pharmacological approaches)

2. Address vision and hearing loss (e.g., ensure glasses and hearing aids are being worn correctly)

3. Sleep at night, not during the day (e.g., increase daily sunlight exposure and decrease night-time noise and light)
4. Find opportunities for creative expression, like art, music, and storytelling

5. Exercise (e.g., in group settings facilitated by videoconference)

6. Maintain religious and cultural practices (e.g., use telephone or videoconference to connect with religious communities)

7. Garden, either indoors or outside

8. Visit with pets (e.g., encourage families to bring animals to window visits)

9. Use technology to communicate (e.g., use phones and tablets to communicate through videocalls, take part in online Resident Forums and Conversation Cafes)

10. Laugh together

11. Reminisce about events, people, and places (e.g., encourage LTC staff to converse with residents at mealtimes)

12. Address communication impairments and communicate non-verbally (e.g., use body language, eye contact, and facial expressions)

These strategies are low risk, familiar, and can be implemented by LTC staff, families, and residents. These strategies can and should be implemented and evaluated in LTC homes right now. While COVID-19 is new, disease outbreaks in LTC homes are not; these strategies may also be used and studied to plan and prepare for the next infectious disease outbreak in LTC.

Some of these strategies rely entirely on technology, or can be adapted through the use of technology, and many LTC homes have enacted their own innovative approaches to care using technology. While technology offers wideranging and exciting opportunities, it has also presented a new set of challenges. ${ }^{(16)}$ Among the key considerations for residents, families, and LTC homes include purchasing new equipment and infrastructure (e.g., costs and set-up time for wireless network, devices and equipment), ensuring scheduled and consistent interactions (e.g., videoconferences with allocated time for each resident), adapting to meet the needs of individual residents (e.g., using television screens instead of phone- or tablet-sized screens), and considering human resource capabilities (e.g., resources to train LTC staff or engage volunteers). Technological solutions should be considered alongside other strategies.

\section{Opportunities to Learn from LTC Homes that Provide Innovative Resident-Centred Care}

COVID-19 has been devastating for LTC residents, families, and staff. As a sector that is already under-valued and underappreciated, ${ }^{(12)}$ it is important to highlight the vital role that LTC homes play in supporting residents and their families. In Canada alone, there are numerous examples of research conducted to reduce disease burden, ${ }^{(17-19)}$ enhance quality of life, ${ }^{(20-22)}$ and improve care experiences ${ }^{(23-25)}$ in LTC homes. While the sector faces challenges, we must also highlight what has been done well. These efforts cannot happen without the support of LTC organizations, LTC staff and managers, 


\section{BETHELL: SOCIAL CONNECTION IS ESSENTIAL IN LTC HOMES}

families, and residents. We must move forward together to reduce exposure to COVID-19 while also enabling and promoting the social connections that are essential to all of us.

\section{ACKNOWLEDGEMENTS}

The authors thank the members of the Alzheimer Society of Canada COVID-19 and Dementia Task Force for their thoughtful comments on the draft manuscript. Jennifer Bethell is supported by the Walter \& Maria Schroeder Institute for Brain Innovation and Recovery. Hannah O'Rourke is supported by an Alzheimer Society Research Program New Investigator Award and a Faculty of Nursing, University of Alberta Professorship in Dementia Care Interventions.

\section{CONFLICT OF INTEREST DISCLOSURES}

The authors declare that no conflicts of interest exist.

\section{REFERENCES}

1. Berkman LF, Glass T, Brissette I, et al. From social integration to health: Durkheim in the new millennium. Soc Sci Med. 2000;51(6):843-57.

2. Leedahl SN, Sellon A, Chapin RK. Assessment of multiple constructs of social integration for older adults living in nursing homes. J Gerontol Soc Work. 2018;61(5):526-48.

3. O'Rourke HM, Collins L, Sidani S. Interventions to address social connectedness and loneliness for older adults: a scoping review. BMC Geriatr. 2018;18(1):1-3.

4. Bradshaw SA, Playford ED, Riazi A. Living well in care homes: a systematic review of qualitative studies. Age Ageing. 2012; 41(4):429-40.

5. Canadian Institute for Health Information. Dementia in longterm care. Ottawa, ON: The Institute; 2018. Available from: https://www.cihi.ca/en/dementia-in-canada/dementia-acrossthe-health-system/dementia-in-long-term-care. Accessed 2020 September 17.

6. O'Rourke HM, Duggleby W, Fraser KD, et al. Factors that affect quality of life from the perspective of people with dementia: a metasynthesis. J Am Geriatr Soc. 2015;63(1):24-38.

7. Moyle W, Kellett U, Ballantyne A, et al. Dementia and loneliness: an Australian perspective. J Clin Nurs. 2011;20(9-10): 1445-53.

8. Bethell J, Aelick K, Babineau J, et al. Social connection in long-term care homes: a scoping review of published research on the mental health impacts and potential strategies during COVID-19. J Am Med Dir Assoc. 2021;22(2):228-37.

9. Kiely DK, Flacker JM. The protective effect of social engagement on 1-year mortality in a long-stay nursing home population. J Clin Epidemiol. 2003;56(5):472-78.

10. Alarcao V, Madeira T, Peixoto-Placido C, et al. Gender differences in psychosocial determinants of self-perceived health among Portuguese older adults in nursing homes. Aging Ment Health. 2019;23(8):1049-56.

11. Puurveen G, Baumbusch J, Gandhi P. From family involvement to family inclusion in nursing home settings: a critical interpretive synthesis. J Fam Nurs. 2018;24(1):60-85.
12. McGilton KS, Escrig-Pinol A, Gordon A, et al. Uncovering the devaluation of nursing home staff during COVID-19: are we fuelling the next health care crisis? J Am Med Dir Assoc. 2020;21(7):962-65.

13. Stall NM, Johnstone J, McGeer AJ, et al. Finding the right balance: an evidence-informed guidance document to support the re-opening of Canadian nursing homes to family caregivers and visitors during the coronavirus disease 2019 pandemic. $\mathrm{J} \mathrm{Am}$ Med Dir Assoc. 2020;21(10):1365-70.

14. Brimelow RE, Wollin JA. Loneliness in old age: interventions to curb loneliness in long-term care facilities. Act Adapt Aging. 2017;41(4):301-15.

15. Quan NG, Lohman MC, Resciniti NV, et al. A systematic review of interventions for loneliness among older adults living in longterm care facilities. Aging Ment Health. 2020;24(12):1945-55. Epub 2019/10/12.

16. Chu $\mathrm{CH}$, Donato-Woodger S, Dainton CJ. Competing crises: COVID-19 countermeasures and social isolation among older adults in long term care [editorial]. $J$ Adv Nurs. 2020; 76(10):2447-80.

17. Boscart V, Davey M, Crutchlow L, et al. Effective chronic disease interventions in nursing homes: a scoping review based on the knowledge-to-action framework. Clin Gerontol. 2020:1-14.

18. Hirdes JP, Major J, Didic S, et al. A Canadian cohort study to evaluate the outcomes associated with a multicenter initiative to reduce antipsychotic use in long-term care homes. $\mathrm{J} \mathrm{Am} \mathrm{Med}$ Dir Assoc. 2020;21(16):817-22.

19. McCracken R, McCormack J, McGregor MJ, et al. Associations between polypharmacy and treatment intensity for hypertension and diabetes: a cross-sectional study of nursing home patients in British Columbia, Canada. BMJ Open. 2017;7(8):e017430.

20. Kehyayan V, Hirdes JP, Tyas SL, et al. Predictors of long-term care facility residents' self-reported quality of life with individual and facility characteristics in Canada. J Aging Health. 2016;28(3):503-29.

21. McGilton KS, Rochon E, Sidani S, et al. Can we help care providers communicate more effectively with persons having dementia living in long-term care homes? Am J Alzheimers Dis Other Demen. 2017;32(1):41-50.

22. Ducak K, Denton M, Elliot G. Implementing Montessori Methods for Dementia ${ }^{\mathrm{TM}}$ in Ontario long-term care homes: recreation staff and multidisciplinary consultants' perceptions of policy and practice issues. Dementia. 2018;17(1):5-33.

23. Kaasalainen S, Hunter PV, Dal Bello-Haas V, et al. Evaluating the feasibility and acceptability of the Namaste Care program in long-term care settings in Canada. Pilot Feasibility Stud. 2020; 6(1):34.

24. Graham ME, Fabricius A. Beyond "home-like" design: visitor responses to an immersive creative space in a Canadian longterm care facility. J Appl Gerontol. 2019;38(7):1045-57.

25. Duggleby W, Ruiz KJ, Ploeg J, et al. Mixed-methods single-arm repeated measures study evaluating the feasibility of a web-based intervention to support family carers of persons with dementia in long-term care facilities. Pilot Feasibility Stud. 2018;4(1):165.

Correspondence to: Jennifer Bethell, PhD, KITE-Toronto Rehabilitation Institute, University Health Network, 550 University Ave., Toronto, ON M5G 2A2

E-mail: Jennifer.bethell@uhn.ca 